\title{
SPIN-FORBIDDEN ELECTRONIC EXCITATIONS IN TRANSITION METAL COMPLEXES
}

\author{
LAWRENCE L. LOHR, Jr. * \\ Department of Chemistry, Unu ersity of Michigan, Ann Arbor Michigan 48107 (US A)
}

(Received July 5th, 1971)

\section{CONTENTS}

$\begin{array}{lr}\text { A Introduction } & 241 \\ \text { B General treatment of spectral intensities } & 242 \\ \text { C. Spin-orbit coupling } & 244 \\ \text { D Effects of spin-orbit coupling on spectral intensities } & 248 \\ \text { E. Exchange coupling and the spectra of ion pairs } & 252 \\ \text { References } & 257\end{array}$

\section{A INTRODUCTION}

A review is presented of recent progress in the detaled understanding of spin-forbidden electronic excitations in transition metal complexes The quantum mechanical description of the role of spin-orbit coupling in such transitions is outlined and related to experimental values of the oscillator strength for the absorption of hight These intensities are shown to be a sensitive measure of certain features in the electronic wave-function Emphasis is placed on the direct couphing of states of diffenng spin by vanous spin-dependent radiative operators. For binuclear and polynuclear complexes there is also the possibility of spinforbidden transitions occurring via an exchange-dependent mechanism rather than via spin-orbit coupling The experimental evidence for this is reviewed and related to recent studies of the coupling between spin excitation waves and electronic excitation waves in magnetically ordered transition metal salts

During the past two decades we have seen a great advance in our understanding of the electronic structure of transition metal complexes ${ }^{1}$ While a variety of expermental techniques have contributed to this progress, a central role has been played by electronic spectroscopy in the visible and UV spectral regions Absorption experments have probably played a greater role than emission studies because the former yield information aboul more excited states than the latter The electronic transitions responsible for the absorption bands frequently involve the rearrangement of electrons within the partially filled $d$ shell of the central ion, although other types, such as ligand-to-metal charge transfer transitions, may be observed The former type, often called a crystal-field transition. is gener-

\footnotetext{
- Alfred P Sloan Reseurch Fellow
}

Coord. Chem Rev., 8 (1972) 
ally weak in intensity because of the parity forbiddeness for an electric dipole process ${ }^{2}$ This restriction is usually overcome by the presence of static non-centrosymmetric components of the crystal field or by a vibronic mechanism involving a suitable non-centrosymmetric vibration. A weak but parity-allowed process such as the magnetic dipole mechanısm may also occur

Many of the more interesting and important crystal field transitions involve states of different total electronic spin ${ }^{3}$ The most famous example is probably the red emission line of the ruby laser, the transition being from the ${ }^{2} E_{g}$ excited state of $\mathrm{Cr}_{\mathrm{r}}$ III $\mathrm{in}$ a nearly octahedral site in $\mathrm{Al}_{2} \mathrm{O}_{3}$ to the ${ }^{4} A_{2 g}$ ground state Since transitions in which the spin changes cannot occur via electric dipole, magnetıc dipole or electric quadrupole mechanisms unless some spin-dependent interaction such as spin-orbit coupling is operative, these transitions, if parity-forbidden as well, are characteristically extremely weak it is the nature of these spin-dependent intensity mechanisms that we wish to explore in this review

\section{B. GENCRAL TREATMENT OF SPECTRAL INTENSITICS}

Spectral absorption intensities can be conveniently expressed ${ }^{4,5}$ in terms of the dimensionless oscillator strength

$$
f=\frac{1000 m c^{2} \ln 10}{N_{0} \pi e^{2}} \int \epsilon(\bar{\nu}) \mathrm{d} \bar{\nu}
$$

where $N_{0}$ is Avogadro's number, $m$ the electron mass, $-e$ the electron charge, $c$ the velocity of light and $\epsilon(\bar{\nu})$ the molar extinction coefficient for light with frequency $\nu=c \bar{\nu}$ For Gaussian line shapes

$$
\int \epsilon(\bar{\nu}) \mathrm{d} \bar{\nu}=(\pi / \ln 2)^{\frac{1}{2}} \epsilon_{0} \delta
$$

where $\epsilon_{0}$ is the maximum extinction coefficient and $\delta$ is the half-wdth in $\mathrm{cm}^{-1}$ at halfheight, so that

$$
f=920 \times 10^{-9} \epsilon_{0} \delta
$$

The semi-classical quantum theory of radiation yields ${ }^{5}$ an expression for $f$ in terms of matrix elements $M_{a b}$ between initial state $a$ and final state $b$, viz

$$
f=\frac{4 \pi m \nu}{\hbar}\left|M_{a b}\right|^{2}
$$

where $\hbar$ is Planck's constant divided by $2 \pi$. For unpolarized light passing through an isotrople medium 


$$
\begin{gathered}
\left|M_{a b}\right|^{2}=\left\{\frac{1}{3}|\langle a|R| b\rangle|^{2}+\left|\left\langle a\left|\frac{1}{2 m c}(L+2 S)\right| b\right\rangle\right|^{2}\right. \\
\left.+\left.\frac{3 v^{2}}{40 c^{2}} \sum_{\alpha \beta}\left\langle a\left|Q_{\alpha \beta}\right| b\right\rangle\right|^{2}+.\right\}
\end{gathered}
$$

Here the terms represent the electric dipole, magnetıc dipole and electric quadrupole contributions, the last having for an operator the traceless quadrupole tensor $Q$ with elements $Q_{\alpha \beta}=r_{\alpha} r_{\beta}-\frac{1}{3} r^{2} \delta_{\alpha \beta}$, where $\delta_{\alpha \beta}$ denotes the Kronecker delta The operators $R, L+2 S$ and $Q$ are one-electron operators, meaning that each is a sum over electrons of one-electron terms, such as $R=\Sigma r_{l}$, where $r_{t}$ is the position vector for the th electron Similarly $(L+2 S)=$ $\Sigma\left(l_{t}+2 s_{t}\right)$ where $l_{t}$ and $s_{t}$ denote operators for the orbital and spin angular monentum of the $t$ th electron

Although no derivation of eqns. (4) and (5) will be given here, it will be important for our later discussion to note that all terms in eqn (5) except that involving the electron spin $\boldsymbol{S}$ are obtained ${ }^{5}$ from the interaction of the electron linear momentum $\boldsymbol{p}$ with the vector potential $\boldsymbol{A}$ of the electromagnetic field The Hamiltonian representing this interaction is a familiar result of time-dependent perturbation theory and is, for a system with $n$ electrons,

$$
H=\frac{e}{m c} \sum_{i=1}^{n} A_{i} \cdot p_{i}
$$

where the second-order term, containing $A_{l}^{2}$, has been omitted. The electnc dipole contribution in eqn. (5) is the term arising from the spatially unform part of the tume-varymg vector potential $A$, the matrix elements of $P \equiv \Sigma p_{2}$ thus required may if desired be converted to matrix elements of $R$ by use of the commutator

$$
P=\frac{m \mathrm{~d} R}{\mathrm{~d} t}=\frac{-1 m}{\hbar}\left[R, H_{0}\right]
$$

where $H_{0}$ is the unperturbed Hamiltonidn of the system, is $\sqrt{ }-1$, and $\left[R, H_{0}\right] \equiv$ $R H_{0}-H_{0} R$ Thus

$$
\langle a|P| b\rangle=\frac{-1 m}{\hbar}\left(E_{b}-E_{a}\right)\langle a|R| b\rangle
$$

where $|a\rangle$ and $|b\rangle$ are eigenstates of $H_{0}$ with energy eigenvalues $E_{a}$ and $E_{b}$ respectively A similar analysis yields the electric quadrupole and orbital magnetic dipole contributions to eqn. (5) in terms of the linear variation of $A$ across the molecule, this variation being very small for wavelengths large relative to the molecule. Of particular importance are the modifications of eqns. (6)-(8) that arise when spin-orbit coupling is important. Finally, the spin magnetic dipole contribution arises from the direct interaction of the spin magnetic moment with the magnetic field of the radiation.

Coord. Chem Rev., 8 (1972) 
For molecules in a medium with index of refraction $n$, each contribution to $f$ must be multiplied by an appropriate factor ${ }^{6}$, which is $\left(n^{2}+2\right)^{2} / 9 n$ for an electric dipole, $n$ for a magnetic dipole, and $n\left(n^{2}+2\right)^{2} / 9$ for an electric quadrupole For visible light and water these factors are 119,133 and 210 , respectively. A suggestion has been made ${ }^{7}$ that otherwise feeble quadrupole transitions in rare earth ions might become considerably enhanced duc to the presence of highly polanzable ligands or solvent molecules The evidence to date for such "pseudo-quadrupolar" transitions does not appear to be conclusive

In general, the spectra of transition metal complexes can be accounted for via an electric dipole mechanism There are a few interesting cases where a magnetic dipole process has been shown to be operative ${ }^{8-10}$, but there is no evidence for an electric quadrupole process Higher multipole processes are even less likely, although the neit section describes their possible role in spin-forbidden transitions For atoms described by RussellSaunders coupling, such that $L, S, M_{L}$ and $M_{S}$ are good quantum numbers, the magnetic moment vector $(L+2 S)$ has only matrix elements diagonal in $L$ and $S$, with $\Delta M_{L}=0, \pm 1$ and $\Delta M_{S}=0, \pm 1$ Further, orthogonality conditions ensure that different multiplets with the same $L$ and $S$ are not connected These selection rules have important consequences for spin-forbidden magnetic dipole transitions such as observed in some $\mathrm{Mn}^{\mathrm{II}}$ complexes

\section{SPIN-ORBIT COUPLING}

The effects ${ }^{11}$ of spin-orbit coupling in our consideration of radiative processes are three-fold

(1) Changes produced in state energies by splittings and/or shifts

(2) Changes produced in wave functions

(3) Changes produced in quantum mechanical operators used for expressing radiative transition rates or oscillator strengths.

The effects on energy levels in terms of first-order Landé splittıngs and second-order shifts are too familar to discuss further ${ }^{12}$. The most important effect on wave functions is to mix states of different spin multiplicity, thus destroying $S$ and $M_{S}$ as good quantum numbers and providing the basis for nearly all treatments of spin-forbidden transitions in atoms and molecules Mizushima ${ }^{13}$ has pointed out that such transitions can also occur without spin-orbit coupling by a suitable higher multıpole transition, such as magnetic quadrupole for $\Delta S=0$ or \pm 1 . even-to-odd transitions, and magnetic octupole for $\Delta S=0$ or \pm 1 , evento-even and odd-to-odd transitions These processes, are, however, expected to be extremely weak and unmportant relative to spin-orbit-dependent processes, except possibly for systems with atoms of very low atomic number ${ }^{14}$

The spin-orbit interaction for an $N$-electron atom with nuclear charge $Z e$ is given ${ }^{15}$ by

$$
H_{\mathrm{SO}}=\frac{e^{2}}{2 m^{2} c^{2}}\left[2 \sum_{l} \frac{l_{l} s_{l}}{r_{l}^{3}}-\sum_{l \neq l}\left(\frac{r_{l} \times p_{l}}{r_{l j}^{3}}\right)\left(s_{l}+2 s_{j}\right)\right]
$$


where the energy is medsured in ergs, $r_{t}$ is the distance in centineters of the $t$ th electron from the nucleus, $r_{i j}$ the distance between the th and $j$ th electrons and $p_{l}$ is the linear momentum operator $-i \hbar \nabla$ for the $i$ th electron. The various spin and orbital angular momentum operators are in units of $\hbar$. The first term, with a summation over all electrons. represents the coupling due to the electric field of the nucleus The second term is summed over all electron pairs, with $I \neq J$ implying the separate inclusion of $I>J$ and $i<J$ Although the Coulomb repulsion does not include separate contributions for these cases $H_{\text {SO }}$ does This can be seen by realizing that the part of the second term containing $s_{i}$ represents the coupling for the $z$ th electron due to the electric field of the $J$ th elfctron. while the part containing $s$, represents the interaction of the orbital magnetic moment of the $i$ th electron with the spin magnetic moment of the $y$ th electron, the corresponding terms given by interchanging $l$ and $l$ are separate contributions and are included in the sumimation. The dipolar spin-spin contribution is also of the orde $\left(m^{2} c^{2}\right)^{-1}$ but has been omitted from eqn (9) since it makes no direct contribution to the spin-oibit coupling constant although it can contribute to devidtions from the Landé interval iule.

It is interesting to note that those terms in eqn (9) representing the coupling of the spin and orbital moments of a given electron due to the electric field of the nucleus and the other electrons are best viewed in the laboratory frame of reference as interactions involving the velocity-dependent electric dipole moment $\boldsymbol{\mu}_{c}$ of the electron These terms are derived ${ }^{15}$ from the more general Hamiltonian

$$
H_{\mathrm{SO}}=\frac{1}{2 m^{2} c^{2}} \sum_{l} s_{l} \times \operatorname{grad}_{l} V p_{l}
$$

Since $-\operatorname{grad}_{l} V$ is the electrostatic force $F_{i}=-c \epsilon_{t}$ acting on the $i$ th electron, where $\epsilon_{l}$ is the electric field, we can write

$$
H_{\mathrm{SO}}=\frac{-e}{2 m^{2} c^{2}} \sum_{l} s_{l} \times p_{l} \varepsilon_{l}=-\sum_{t} \mu_{e_{l}} \varepsilon_{l}
$$

where the electric dipole moment of the electron is $\left(e / 2 m^{2} c^{2}\right) s \times p$. For an electron moving across this page from left to right with spin "up" (out of the page), the electric moment is directed toward the top of the page. meaning that the electron is more negative toward the bottom of the page This is an energetically stable situation it there is a positive charge (the nucleus) toward the bottom of the page (Fig I, left side) I1 instead the spin is "down", the direction of the electric moment is reversed, creating an energetically unstable situation if there is. as before, a positive charge toward the botton of the page (Fig 1, right side) It should be noted that the dipole moment of a charged species is origin-dependent and can thus have any value, including zero if the origin is tahen at the center of charge Since linear momentum is origin-independent, the origin for the dipole moment in eqn (11) appears to be the origin for the spill angular momentum $s$

Following the analysis of Blume and Watson ${ }^{15}$, based on the earher work of Horte ${ }^{16}$, consider an atom or ion with a single unfilled shell outside a number of closed sinells, as is the case with transition metal ions The nuclear term in eqn (9) will contain a s.mmation 


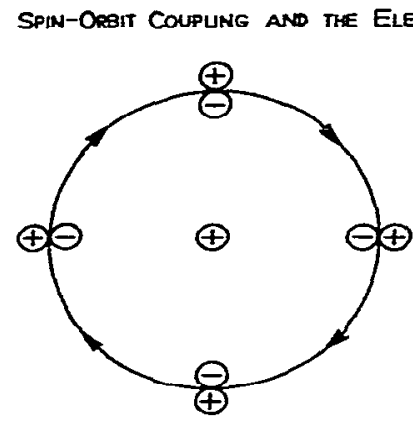

(a)

SPIN ANGULAR MOMENTUM UP ORBITAL " " DOWN EnERgetically Stable

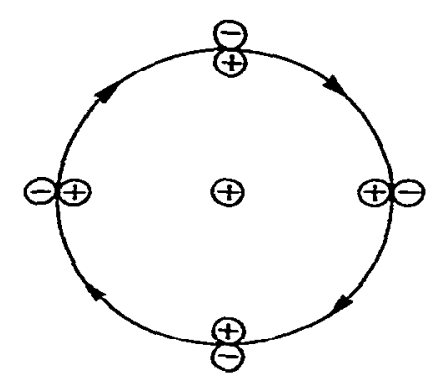

(b)

Fig 1 An illustration of spin-orbit coupling tor an electron in the field of a positive charge in terms of the relativistic electric dipole moment of the electron (eqn. (11)) The positive charge is at the center of the circle and the motion is taken clock wise for (a) spin angular momentum up, electric moment pointing dway trom nucleus and (b) spin angular momentum down, electric moment pointing toward nucleus The electron charge is not shown, only the dipole moment

over electrons in the closed shells and a summation over electrons in the open shell The first group makes no net contribution to the energy due to a cancellation of positive and negative terms so that nuclear contributions to the spin-orbit energy arise only from the open shell electons. The summation over electron pairs in the second term of eqn (9) will consist of three parts (a) terms from electrons of which both are in either the same or different closed shells, $(b)$ terms from electrons one of which is in the open shell, $(c)$ terms from electrons of which both are in the open shell The sum of all contributions of the first type will be zero, again by a cancellation of positive and negative contributions The sum over closed shells of all contributions of the second type for a given outer shell electron yields a contribution in form like that from the nucleus but of opposite sign, thus representing a screening of the nuclear charge which acts to reduce the coupling constant We can then write

$$
H_{\text {SO }}=\xi_{c} \sum_{l}^{\text {outer }} I_{l} s_{l}-\frac{e^{2}}{2 m^{2} c^{2}} \sum_{l \neq j}^{\text {outer }}\left(\frac{r_{l j}}{r_{l j}^{3}} \times p_{l}\right)\left(s_{l}+2 s_{l}\right)
$$

The quantity $\zeta_{C}$ thus represents the strength of the spin-orbit coupling for an outer electron in the Coulomb field of a nucleus shielded by closed inner shells

It has been shown that a part of the second term, representing interactions between electrons that are both in the unfilled shell, can be represented as an effective one-electron coupling Modifying $\zeta$ to include this part of the second term, and then ignoring the residual two-electron contributions, which do not contain additive terms whose matrix elements are proportional to $\Sigma l_{l} s_{l}$, we obtain 


$$
H_{\mathrm{SO}}=\xi^{\prime} \sum_{t}^{\text {outer }} l_{1} s_{t}
$$

The residual interactions omitted in eqn (13) do, however, contribute as much as $10 \%$ of the total of an interaction assumed to be of the form $\lambda \boldsymbol{L} \cdot S$, which describes first-order (aragonal) spin-orbit splittings in Russell-Saunders coupling. Such a contribution indicates the magnitude of the error in obtaining "experimental" values $\zeta$ ' from the Lande $\lambda$ via the relation $\zeta=2 S|\lambda|$, particularly when $\zeta^{\prime}$ and eqn (13) are used to evaluate offdiagonal matrix elements $A$ theoretical value of $\lambda$ may be obtained ${ }^{15}$ from diagonal matrix elements of eqn. (12) in a Russell-Saunders basis, thus including all two-electron terms correctly to first-orcer

Some exdmple of the values (all in $\mathrm{cm}^{-1}$ ) computed ${ }^{15}$ from atomic SCF radial functions are listed in Tables 1 and 2 . For Sc II , $3 d^{\mathbf{1}}$, with no summation over outer electrons, the values of $\zeta_{c}, \zeta^{\prime}$ and $\lambda$ are identical For $M n^{\prime \prime}, 3 d^{5}, \lambda$ is not computed as no first-order Landé splitting is observed for the half-filled shell For $\mathrm{Cu}$ II $, 3 d^{9}, \zeta_{c}=866, \zeta^{\prime}=826$ and $\lambda=-830$, showng that the single hole differs from a single electron Althuugh the difference between $\xi^{\prime}$ and $2 S|\lambda|=|\lambda|$ for $\mathrm{Cu}$ II is small, the difference is greater for $\mathrm{Cr}$ III, $3 d^{3}$ with $\zeta^{\prime}=292$ and $\lambda=91(2 S|\lambda|=3 \lambda=273)$

If a molecule is considered instead of an atom, the spin-orbit Hamiltonian is similar to eqn. (9), but with a nuclear contribution that is

$$
H_{\mathrm{SO}}(\text { nuclear })=\frac{e^{2}}{2 m^{2} c^{2}} \sum_{K} Z_{K} \sum_{t} \frac{l_{I K} \cdot s_{1}}{r_{l K}^{3}}
$$

\section{TABLE 1}

\begin{tabular}{|c|c|c|c|c|c|c|c|}
\hline Ion & Contiguration & $\zeta$ (nuclear) & $\zeta_{c}$ & $\xi^{\prime}$ & $\lambda$ & $\Delta \xi^{c}$ & $\lambda(\mathrm{obs})^{d}$ \\
\hline scll & $3 d^{1}$ & 193 & 857 & 857 & 857 & 0 & 79 \\
\hline$T_{4}^{I I}$ & $3 d^{2}$ & 275 & 126 & 126 & 61 & 4 & $59-61$ \\
\hline$v^{11}$ & $3 d^{3}$ & 370 & 186 & 184 & 57 & 13 & 56 \\
\hline $\mathrm{Cr}^{\mathrm{II}}$ & $3 d^{4}$ & 484 & 262 & 258 & 59 & 22 & $54-61$ \\
\hline $\mathrm{Mn}^{I I}$ & $3 d^{5}$ & 622 & 342 & 333 & & & \\
\hline$\Gamma_{\mathrm{e}} \mathrm{II}$ & $3 d^{6}$ & 773 & 440 & 426 & -114 & 30 & -94 to -109 \\
\hline Coll & $3 d^{7}$ & 953 & 560 & 539 & -189 & 28 & -166 to -186 \\
\hline $\mathrm{Ni}^{\text {II }}$ & $3 d^{8}$ & 1162 & 702 & 672 & -343 & 14 & -303 to -340 \\
\hline$C u^{I I}$ & $3 d^{9}$ & 1399 & 866 & 862 & -830 & 4 & -829 \\
\hline
\end{tabular}

Spin-orbit coupling paramcters ${ }^{a} b$ trom SCI radial functions for divalent transition metal ions

a All values are in $\mathrm{cm}^{-1}$ and taken trom ret (15)

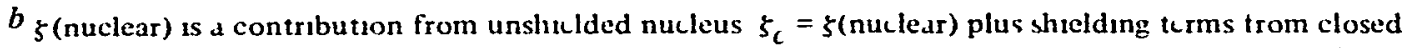
inner shells, $\xi^{\prime}=\zeta_{c}$ plus shielding terms from other $d$ electrons, while $\lambda$ is obidined trom disgond matrix elements of eqn (12)

$c \Delta \zeta \equiv \zeta-2 S|\lambda|$

$d$ Observed values tor free tons 
TABLE 2

Spin-orbit coupling parameters ${ }^{a}$ from SCF radial functions for trivalent transition metal ions

\begin{tabular}{|c|c|c|c|c|c|c|c|}
\hline Ion & Configuration & $\xi$ (nuclear) & $\xi_{c}$ & $\xi^{\prime}$ & $\lambda$ & $\Delta \zeta^{b}$ & $\lambda$ (obs) \\
\hline $\mathrm{Tl}^{\mathrm{III}}$ & $3 d^{1}$ & 328 & 159 & 159 & 159 & 0 & 154 \\
\hline $\mathrm{V}^{\text {III }}$ & $3 d^{2}$ & 433 & 220 & 219 & 106 & 7 & $104-106$ \\
\hline CrIII & $3 d^{3}$ & 556 & 296 & 292 & 91 & 19 & $88-97$ \\
\hline $\mathrm{Mn}^{\mathrm{III}}$ & $3 d^{4}$ & 700 & 388 & 380 & 87 & 32 & $83-92$ \\
\hline$\Gamma c^{111}$ & $3 d^{5}$ & 871 & 499 & 486 & & & \\
\hline
\end{tabular}

$a$ All values are in $\mathrm{cm}^{-1}$ and are taken from ref. (15)

$b_{\Delta \xi}=\xi^{\prime}-2 S I \lambda J$

where $r_{i K}$ is the distance of the $i$ th electron from the $K$ th nucleus with charge $Z_{K} e, l_{t K}$ is the operator for orbital angular momentum of the $t$ th electron about the $K$ th nucleus and the summation is over both electrons and nuclei The spin-orbit coupling in a molecule can then be approximated by an effective one-electron interaction of the form in eqn. (13) but containing a nuclear contribution as in eqn (14) It should be noted that $\zeta$ (nuclear) for a molecule is different from that for an atom even if the wave functions are assumed to be the same. as in a crystal field model of transition metal complexes Thus even with purely $3 d$ orbitals, eqn (14) involves a summation over ligand nuclei in addition to the central ion nucleus, although the ligand contributions are expected to be small because of the $\left(r_{1 K}\right)^{-3}$ factors when the electron is centered on the metal ion and $K$ is a ligand nucleus More important, however, is the fact that the total orbital angular momentum is not a good quantum number for molecules

\section{EFFECTS OT SPIN-ORBIT COUPLING ON SPECTRAL INTENSITIES}

We have pointed out ${ }^{17}$, as have others ${ }^{11,18,19}$, that the dipole length operator $R$ is valid for describing electric dipole intensities in systems where spin-orbit coupling is important However, if a representation in terms of momentum matrix elements is desired instead, the correct operator, when eqn (13) holds, is

$$
\pi=\sum_{i} \pi_{l}=\sum_{l}\left[p_{l}+\frac{1}{2 m c^{2}} s_{l} \times \operatorname{grad}_{l} V\right]
$$

where the summation is over electrons. $p$, is the linear momentum operator for the $i$ th electron, and-grad, $V$ is the electrostatic force acting on the $\imath$ th electron The transformation to matrix elements of $R$ follows from the commutation relation

$$
\pi=m \frac{\mathrm{d} R}{\mathrm{~d} t}=\frac{-1 m}{\hbar}[R, H]
$$

where the total Hamiltonian $H$ contains $H_{\text {SO }}$ as in eqn (13) (If $V$ in eqn (15) is identified with the Hartree potential energy in a many-electron atom, our identification of $\pi$ with 
$H_{\text {SO }}$ in eqn. (13) should be made instead with the Watson-Blume parameter $\zeta_{d}^{\prime}=$ $\left(e^{2} / 2 m^{2} c^{2}\right)\langle\partial V / r \partial r\rangle$, which does not contain exchange contributions.)

Since $\pi$ is in part spin-dependent, the operator can connect basis states of different spin Thus in the momentum operator representation of the intensity, the spin-orbit coupling need not mix states in order to make spin-forbidden transitions allowed Frequently the question is asked from what spin-allowed transition is the intensity borrowed for a spinforbidden transition? It is important to realize that although the question is sometimes useful, the answers are artificial, for they depend not only on the basis ser chosen to represent the unperturbed states having an allowed transition, but also upon the operator for the radiative process An illustration of this dependence is given in the next section

Chu ${ }^{19}$ has made a thorough analysis of the operators for radiative transitions and has concluded that all relativistic effects, non-conservative electromagnetic forces derivable from a vector potential, non-Hermitian terms, corrections due to small-component spinors. etc, can be grouped together into an "effective" transition momentum $\pi^{\text {ett }}$, which in general is different from the linear momentum $p_{l}$, but which like $\pi$ in eqn. (15) is related to $m(\mathrm{~d} R / \mathrm{d} t)$ and to $R$ via eqn (16), thus providing the generalization of our result. His analysis explicitly considers the dipolar spin-spin interaction, spin-other-orbit coupling, as well as the direct interaction of the spin with the magnetic part of the radiation field The latter interaction, not being derivable from a vector potential. must be included as a separate transition operator Chiu gives an extensive listing of spin-dependent operators for direct $\Delta S= \pm 1$ radiative transitions, together with numerical estimates of their importance relative to the usually considered indirect spin-orbit processes involving the mixing of wave functions of differing spin These operators arise from the following interactions

(a) Spin-own-orbit effect, identical to the spin part of $\pi$ (eqn (15)), having odd spatial parity, and typical size ${ }^{21}$ (matrix element squared) of $88 \times 10^{-33}$ for direct $\Delta S= \pm 1$ transition

(b) "Ordinary" spin radiation coupling, having odd spatial parity and typical size of $86 \times 10^{-37}$

(c) Correction for small-component spinors, having odd spatial parity and typical size of $21 \times 10^{-37}$

(d) Spin-own-orbit effect, having even spatial parity, like the term in ret 17 , but without the symmetrization to separate magnetic dipole part from electric quadrupole part, and typical size of $81 \times 10^{-39}$

(e) Spin-other-orbit coupling, having even spatial parity, and typical size of $61 \times 10^{-40}$ By comparison he estmates the typical size of the usual indirect transition strength with odd parity operator to be $21 \times 10^{-31}$ (based on spin-orbit matrix element of $100 \mathrm{~cm}^{-1}$ and energy gap of $10^{5} \mathrm{~cm}^{-1}$ ), while that with even parity operator ${ }^{20}$ is $19 \times 10^{-37}$

As an example consider a transition from the mostly ${ }^{6} S_{\frac{5}{2}}$ ground state $t_{0}$ of a gaseous

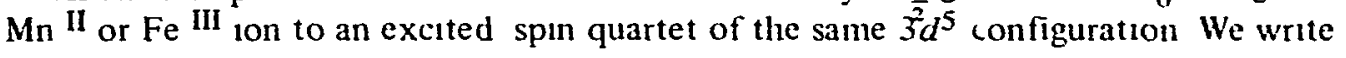

$$
\star_{0}={ }^{6} S_{\frac{5}{2}}+\alpha{ }^{4} P_{\frac{5}{2}} \quad \alpha=-\frac{\left\langle{ }^{4} P_{\frac{5}{2}} \mid H_{S O}{ }^{6}{ }^{6} S_{\frac{5}{2}}\right\rangle}{E\left({ }^{4} P_{\frac{5}{2}}\right)-E\left({ }^{6} S_{\frac{5}{2}}\right)}
$$

Coord. Chem Rev., 8 (1972) 
where the effect of $\alpha$ on the normalization is ignored. Here $\alpha=1-5^{\frac{1}{2}} \zeta /(7 B+7 C)=-2.6 \times 10^{-2}$ for $\zeta=350 \mathrm{~cm}^{-1}$ and repulsion parameters of $B=960 \mathrm{~cm}^{-1}$ and $C=3325 \mathrm{~cm}^{-1}$ The spin-orbit coupling operator $H_{\mathrm{SO}}$ is taken here in the form of eqn. (13) Electric dipole transitions are forbidden, but there is a spin-orbit allowed magnetic dipole transition to the mostly ${ }^{4} P_{\frac{5}{2}}$ state $F$, where

$$
F_{1}={ }^{4} P_{\frac{5}{2}}-\alpha{ }^{6} S_{\frac{5}{2}}
$$

thus

$$
\left.\left\langle\mathcal{F}_{0}|L+2 S| F_{1}\right\rangle=\alpha{ }^{4} P_{\frac{S}{2}}|L+2 S|^{4} P_{\frac{5}{2}}\right\rangle-\alpha\left\langle{ }^{6} S_{\frac{5}{2}}|L+2 S|^{6} S_{\frac{5}{2}}\right\rangle
$$

For the specific component of the ground state with $M_{S}=M_{J}=-\frac{5}{2}$, the magnetic moment of ${ }^{6} S_{\frac{5}{2}},-\frac{5}{2}$ is $-5 \hbar$, that of ${ }^{4} P_{\frac{5}{2}},-\frac{5}{2}$ is $-4 \hbar$, so that, dropping terms in $\alpha^{2}$, we have for the $z$ component, with $\Delta J=\Delta M_{J}^{2}=0,\left\langle x_{0}|L+2 S| x_{1}\right\rangle=\alpha \hbar$ In this atomic example there is also the $\Delta J=-1$ magnetic dipole transition from ${ }^{6} S_{\frac{5}{2}}$ to ${ }^{4} P_{\frac{3}{2}}$, but no transitions in this order of perturbation theory to ${ }^{4} D,{ }^{4} F,{ }^{4} G$, or to any of the spin doublets of $3 d^{5}$

Considering next a tetrahedral Mn II or Fe III complex, such that the parity forbiddeness for electric dipole processes is overcome, we write

$$
{ }^{F_{0}}={ }^{6} A_{1}+\sum_{j=1}^{3} \alpha_{j}{ }^{4} T_{t}^{\prime}
$$

where the summation is over the three cubic field ${ }^{4} T_{1}$ eigenstates, each in turn taken as superpositions of either strong field $\left(t_{2}{ }^{4} e, t_{2}{ }^{3} e^{2}\right.$ and $\left.t_{2}{ }^{2} e^{3}\right)$ or weak field $\left({ }^{4} P,{ }^{4} F\right.$ and $\left.{ }^{4} G\right)$ basis states, and the $\alpha$, are analogous to $\alpha$ in eqn (17) Ignoring all other spin-orbit mixings except those involving the ground state,

$$
\left\langle *_{0}|R| \Psi_{1}\right\rangle=\sum_{l} \alpha_{j}\left\langle{ }^{4} T_{1}^{J}|R|{ }^{4} \Gamma\right\rangle
$$

for $\Gamma=A_{2}, E$ or $T_{2}$ Since $R$ transforms as $T_{2}$ in the group $T_{d}$, the transition to ${ }^{4} A_{1}$ is forbidden in this order For transitions to a mostly ${ }^{4} T_{1}^{J}$ state $f_{2} J$, where

$$
F_{2} I={ }^{4} T_{1}^{J}-\alpha_{j}{ }^{6} A_{1}
$$

we have

$$
\left\langle f_{0}|R| f_{2}{ }^{\prime}\right\rangle=\sum_{l \neq j} \alpha_{j}\left\langle{ }^{4} T_{1}{ }^{i}|R|^{4} T_{1}{ }^{j}\right\rangle
$$

where we have used the fact that diagonal elements of $R$ are zero in symmetry $T_{d}$.

We can also write 


$$
\left\langle x_{0}|\pi| x_{1}\right\rangle=\left\langle{ }^{6} A_{1}|\pi|^{4} \Gamma\right\rangle+\sum_{J} \alpha_{j}\left({ }^{4} T_{1} J|\pi|^{4} \Gamma\right\rangle
$$

where the spin-dependent part of $\pi$ directly connects ${ }^{6} A_{1}$ to ${ }^{4} \Gamma$, but only for $\Gamma=T_{2}$ in group $T_{d}$, the expression for transitions to mostly ${ }^{4} T_{1}$ states will be like eqn. (21), but with $\pi$ replacing $R$.

The magnetic dipole matrix elements, identical for $T_{d}$ and $O_{h}$ symmetries if purely $3 d$ wave functions are assumed (the latter symmetry having no electric dipole matrix elements for $d-d$ transitions barring vibronic mechanisms) are readily determined as

$$
\left\langle f_{0}|L+2 S| f_{1}\right\rangle=\sum_{j=1}^{3} \alpha_{j}\left\langle{ }^{4} T_{1}{ }^{J}|L|^{4} \Gamma\right\rangle
$$

for $\Gamma=A_{1}, E$ and $T_{2}$, with the transition to ${ }^{4} A_{2}$ forbidden, and where the spatially independent $S$ does not connect different spatial states For $\Gamma=T_{1}$

$$
\begin{aligned}
& \left.\left\langle t_{0}|L+2 S| t_{2}{ }^{\jmath}\right\rangle=\alpha_{j} \mid\left\langle{ }^{4} T_{1}{ }^{l}|L+2 S|{ }^{4} T_{1}^{J}\right\rangle-\left\langle{ }^{6} A_{1}|2 S|{ }^{6} A_{1}\right\rangle\right] \\
& \quad+\sum_{l \neq j} \alpha_{l}\left\langle{ }^{4} T_{1}{ }^{l}|L|{ }^{4} T_{1}{ }^{j}\right\rangle+\text { terms in } \alpha^{2}
\end{aligned}
$$

A detalled treatment of the magnetic dipole spectrum of octahedral $\mathrm{Mn}$ II complexes has been given ${ }^{4,10}$, in which the above expressions are modified to include effects of the orthorhombic crystalline fields and exchange field $\mathrm{n}$ antiferromagnetic $\mathbf{M n F}_{2}$

In summary, the types of contributions of the matrix elements for either electric dipole or magnetic dipole spin-forbidden transitions are $(a)$ a term proportional to the product of a spin-orbit mixing coefficient and the diagonal electric or magnetic moment of the initial unperturbed state, (b) a term as in (a), but for the final unperturbed state, $(c)$ a term proportional to the product of a spin-orbit mixing coefficient times an off-diagonal electric or magnetic moment involving the initial unperturbed state, $(d)$ a term as in $(c)$, but for the final unperturbed state, $(e)$ a term involving the direct coupling of the unperturbed states of differing spin

Our examples have illustrated all of these except $(d)$ which for $\mathrm{Mn}$ II complexes might involve the spin-orbit mixing of spin sextets other than the ${ }^{6} S$ of $3 d^{5}$ into the excited quartets Such sextets might arise from charge transfer configurations or higher energy atomic configuration as $3 d^{4} 4 s^{1}$ Although the spin-orbit coupling in a many-electron atom or molecule is approximately represented by eqn (13), several studies ${ }^{13,19}$ have indicated the importance of explicit consideration of the two-electron contributions, both in terms of the perturbation of wave functions and of the effect on the radiative operators for $\Delta S= \pm 1$ transitions. This conclusion is not surprising since the two-electron terms are known to be very important in determining the line structure in gaseous $d^{5}$ ions ${ }^{22}$ and the spectral intensities for $\pi \rightarrow \pi^{*}$ transitions in aromatic hydrocarbons ${ }^{23,24}$.

It is perhaps useful to point out the close simila-ity of the spin-forbidden transitions 
in tons with the half-filled configuration $d^{5}$ to those for the configuration $p^{3}$, exemplified by $\mathrm{N}$ or $\mathrm{O}^{+1}$ the latter being particularly important in gaseous nebulae such as in Orion The doubly forbidden emissions involving the ${ }^{4} S^{0} \frac{3}{2}$ ground state are

$$
{ }^{2} D^{0} \frac{3}{2}\left(\frac{5}{2}\right) \rightarrow^{4} S^{0} \frac{3}{2} \text { and } 2 P^{0} \frac{1}{2}\left(\frac{3}{2}\right)^{4} S^{0} \frac{3}{2}
$$

The transitions from $2 P^{0}$ become magnetic dipole and electric quadrupole allowed by simple spin-orbit mixing. but not those from ${ }^{2} D^{0}$, which are sitisfactorily accounted for ${ }^{25}$ only when the perturbation of the wave functions by the spin-spin and spin-other orbit interactions is considered

In summary we see that the principal characteristics of spin-forbidden transitions induced by spin-orbit coupling are their weakness relative to spin-allowed transitions, but with a strength strongly dependent upon the atomic numbers of the atoms in the molecule The latter is often called the "heavy atom" effect, and is very familiar to spectro. scopists of substituted $\pi$-electron systems 13

An aspect of the coupling that has now recerved thorough theoretical treatment ${ }^{19}$ but only limited application so far to the interpretation of spectra is the direct coupling of states of diftering spin by those spin-dependent radiative operators appropriate to systcms with spin-orbit interactions Finally we shall not review in detal the spectra of individual ions or compleses, but instead refer the reader to the excellent reviews listed under ref 1

\section{EXCHANGE COUPLING AND THE SPLCTRA OF ION PAIRS}

Whereas spin-orbit coupling is necessary for electric or magnetic dipole radiative transitions between electronic states of differing spin of either gaseous ions or mononuclear complexes. there is another mechanism possible for binuclear complexes and larger aggregrates of paramagnetic ions, including infinite solid arrays This mechanism is a manifestation of the exchange interaction so familiar from its magnetic consequences However, the implications for spectral intensities have only recently become appreciated and understood. It should be recalled that the exchange interaction is basically electrostatic rather than magnetic in its nature, and that it frequently involves ${ }^{26}$ not only the two-electron "exchange" integral itself, but also contributions from electron kinetic energy, electron nuclear attraction and differences in two-electron Coulomb integrals

Exchange coupled ion parrs in crystals were first observed optically by Schawlow et al 27 for $\mathrm{Cr}$ III $\mathrm{in} \mathrm{Al}_{2} \mathrm{O}_{3}$ They demonstrated the concentration dependence of certain satellite lines in the red emission from the ${ }^{2} E$ state to the ${ }^{4} A_{2}$ ground state.

Later McClure ${ }^{28}$ observed the absoiption by $\mathrm{Mn}$ II pairs in $\mathrm{ZnS}$ Although some of the structure attributed to parrs appears to be $\mathrm{ZnS}$ phonon sidebands instead ${ }^{29}$, 30 , the concentration studies did indicate one of the most important spectral features of ion pairs. namely a pronounced intensification by the exchange interaction for those transitions that are spin-forbidden in isolated ions or mononuclear complexes This property was then clearly demonstrated by concentration studies ${ }^{31}$ of $\mathrm{Mn}{ }^{11}$ in $\mathrm{KZnF}_{3}$, in which the absorp- 
tion intensities per ion for the pair spectra are comparable to those for pure $\mathrm{KMnF}_{3}$, but an order of magnitude greater than those for single Mn II ions in very low concentration (1 2 mole \%) in $\mathrm{KZnF}_{3}$. A similar intensification was observed ${ }^{32}$ by a correlation of intensity to crystal structure for a wide variety of pure $\mathrm{Mn}$ II salts The intensity is reldtively great whenever the $\mathrm{Mn}$ II ions are separated by a single ligand atom as in the obvious cases of $\mathrm{MnS}(\mathrm{NaCl}$ structure $)$ and $\mathrm{MnCl}_{2}\left(\mathrm{CdCl}_{2}\right.$ structure), and in the less obvious case of $\mathrm{MnCO}_{3}\left(\mathrm{CaCO}_{3}\right.$ structure $)$ where one oxygen of a $\mathrm{CO}_{3}{ }^{2-}$ unit serves as a bridge. Other bridgings, as via O-S-O of $\mathrm{SO}_{4}{ }^{2-}$ in $\mathrm{MnSO}_{4} \cdot 4 \mathrm{H}_{2} \mathrm{O}$, are less effective. The intensity is relatively weak for pure salts having discrete complexes, such as cis- $\mathrm{Mn}\left(\mathrm{OH}_{2}\right)_{4} \mathrm{Cl}_{2}$ in $\mathrm{MnCl}_{2}-4 \mathrm{H}_{2} \mathrm{O}$, trans- $\mathrm{Mn}_{2}\left(\mathrm{OH}_{2}\right)_{2} \mathrm{Cl}_{4}{ }^{2-}$ in $\mathrm{Cs}_{2} \mathrm{MnCl}_{4} 2 \mathrm{H}_{2} \mathrm{O}$, and $\mathrm{Mn}\left(\mathrm{OH}_{2}\right)_{6}{ }^{2+}$ in $\mathrm{Mn}\left(\mathrm{ClO}_{4}\right)_{2} 6 \mathrm{H}_{2} \mathrm{O}$ or $\mathrm{MnSiF}_{6} \quad 6 \mathrm{H}_{2} \mathrm{O}$ The presence or absence of a center of inversion symmetry at the $\mathrm{Mn}^{\mathrm{Il}}$ site affects the intensity to a lesser extent than the presence of nearby $\mathrm{Mn}^{\mathrm{II}}$ lons, thus supporting the hypothesis of an exchange-dependent intensity mechanism

Similar intensifications have been found for $\mathrm{N}_{\mathrm{I}}{ }^{\mathrm{II}}$ pairs ${ }^{33}$ and mixed $\mathrm{N}_{1}{ }^{\text {II }}-\mathrm{Mn}$ II pairs ${ }^{34-36}$ in the fluoride perovskites provided that one monitors the spin-forbidden transitions of either the $\mathrm{N}_{1}$ II or Mn II lons However, the spin-allowed crystal-field transitions of $\mathrm{N}_{1}$ II obey Beer's law

The understanding of the nature of the exchange-dependent intensity mechanism has come largely from the many recent detaled studies of the spectra of antiferromagnetic salts such as $\mathrm{MnF}_{2}$ and $\mathrm{RbMnF}_{3}$ In such materials the deviation in the orientation of the spin of an ion in its ground electronic state from the orientation preferred by the cooperative exchange interactions can propagate through the lattice as a spin-wave, with such an excitation called a "magnon" There are three related processes that have been observed to occur in $\mathrm{MnF}_{2}$ and similar materials

(a) The far infra-red $\left(\sim 100 \mathrm{~cm}^{-1}\right)$ electric dipole absorption leading to the creation of two magnons for each photon $37-41$

(b) The visible and UV electric dipole absorption leading to the creation of one magnon and one exciton (an electronic excitation) for each photon $\$ 2-50$.

(c) The UV electric dipole absorption leading to the creation of two excitons for each photon This is described as a two-ion excitation and is revealed by the presence of electronic overtone and combination bands with energies given closely but not exactly by the appropriate sum of the single-ion excitation energies $51-53$

Antiferromagnets such as $\mathrm{MnF}_{2}$ can be described in terms of a two sub-lattice model, one sub-lattice with "up" spins, and the other with "down" spin Neutron diffraction of tetragonal $\mathrm{MnF}_{2}$ (rutile structure) reveals this type of magnetic ordering. with each ion with spin "up" and parallel to the crystal $c$ axis being surrounded by eight ions with spin "down", but also parallel to $c$ There are also two intrd-sub-lattice neighbors of the same spin along the $c$ axis. Letting $A$ denote an ion of one sub-lattice and $B$ a neighboring ion on the second sub-lattice, we see that each of the processes listed above is basically a twoion process, with similar but not identical spin selection rules, as shown in Table 3 The two-magnon excitation, in which the total spin of each ion is unchanged, consists of an

Coord. Chem, Rev., 8 (1972) 
TABLE 3

Spin selection rules for exchange-dependent excitations. ${ }^{a}$

\begin{tabular}{|c|c|c|c|c|c|c|}
\hline & $\Delta s_{A}$ & $\Delta M_{\mathrm{A}}$ & $\Delta S_{\mathrm{B}}$ & $\Delta M_{\mathrm{B}}$ & $\Delta S$ & $\Delta M$ \\
\hline Tho-magron & $\mathbf{o}$ & $\therefore 1$ & 0 & $\mp 1$ & $\mathbf{o}$ & 0 \\
\hline Exciton + magnon $b$ & \pm 1 & \pm 1 & 0 & $\mp 1$ & 0 & 0 \\
\hline Tuoesciton & \pm 1 & \pm 1 & \pm 1 & $\mp 1$ & 0 & $\mathbf{0}$ \\
\hline
\end{tabular}

${ }_{b}^{a} S \equiv S_{\mathrm{A}}+S_{\mathrm{B}}, M \equiv M_{\mathrm{A}}+M_{\mathrm{B}}$, where $M$ is the elgenvalue of $S_{z}$

$b$ Ion $\mathrm{A}$ arbitrarily selected to have the electronic excitation

increase in the $z$-component of the spin for one ion coupled to a decrease for the other, such that the $\operatorname{sum} M=M_{\mathrm{A}}+M_{\mathrm{B}}$ is unchanged Specifically for $\mathrm{Mn}^{\text {II }}$, we have

Sub-lattice A $S_{\mathrm{A}}=\frac{5}{2}, M_{\mathrm{A}}=+\frac{5}{2}, S_{\mathrm{A}}=\frac{5}{2}, M_{\mathrm{A}}^{\prime}=+\frac{3}{2}$

Sub-lattice B $S_{\mathrm{B}}=\frac{5}{2}, M_{\mathrm{B}}=-\frac{5}{2}, S_{\mathrm{B}}=\frac{5}{2}, M_{\mathrm{B}}^{\prime}=-\frac{3}{2}$

where $\Delta S=\Delta M=0$ The initial values of $M$ of $+\frac{5}{2}$ and $-\frac{5}{2}$ for the two sub-lattices reflect the difference in the orientation of the spins Further detalls of pure magnon spectra lie outside the scope of this article.

The exciton plus magnon process is identical to the above with respect to the $M$ quantum numbers, but the total electronic spin of one ion changes, corresponding to a spin-forbidden electronic excitation of that ion It should be emphasized that such an excitation is spinforbidden only for the single on or mononuclear complex $\left(\Delta S_{\mathrm{A}}= \pm 1\right)$, but not for the pair That is, we see in Table 3 that $\Delta S=\Delta M=0$ for this process just as for the two-magnon process. For $\mathrm{Mn}^{\text {II }}$,

Sub-lattice A. $S_{\mathrm{A}}=\frac{5}{2}, M_{\mathrm{A}}=+\frac{5}{2}, S_{\mathrm{A}}^{\prime}=\frac{3}{2}, M_{\mathrm{A}}=+\frac{3}{2}$

Sub-lattice B $S_{\mathrm{B}}=\frac{5}{2}, M_{\mathrm{B}}=-\frac{5}{2}, S_{\mathrm{B}}^{\prime}=\frac{5}{2}, M_{\mathrm{B}}=-\frac{3}{2}$

The possible initial values of $S$ are given by the vector sum of $S_{\mathrm{A}}$ and $S_{\mathrm{B}}$, and are $0,1,2,3,4$ and 5 , while the possible final values of $S$ are given by the vector sum of $S_{A}{ }^{\prime}$ and $S_{\mathrm{B}}$, and are $1,2,3,4$. Thus transitions from any initial pair spin state other than $S=0$

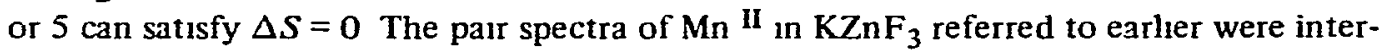
preted ${ }^{31}$ in terms of this selection rule. Such a pair process corresponds in the magnetically ordered infinite array to the creation of an exciton on one magnetic sub-lattice and the creation of a magnon on the other sub-lattice (or the destruction of both in the corresponding emission process). By contrast the creation or destruction of an exciton and a magnon on the same sub-lattice results in change of \pm 2 in the $M$ quantum number and is hence a forbidden process, as weak if not weaker than the very weak magnetic dipole pure exciton transitions that are observed ${ }^{10,54}$ near the origin of the strong exciton-magnon 
sidebands in $\mathrm{MnF}_{2}$. Other $\Delta M=0$ processes which are observed are the two-sub-lattice exciton creation with magnon destruction (absorption hot bands) at suitably elevated temperatures ${ }^{55}$ (approximately $30^{\circ} \mathrm{K}$ ) and the corresponding emission process of exciton destruction and magnon creation, which leaves the system magnetically excited. Sell ${ }^{\text {so }}$ has reviewed the experimental observations for various magnetically ordered salts of $\mathrm{Cr}$ III, Mn II, Fe II, Co II and NI II

There have been a number of detaled theoretical treatments of the cooperative intensity mechanism $37,41,49,56-59$. Several of the studies have been concerned with selection rules $41,49,58$ appropriate to the magnetically ordered crystal, others have considered the nature of the coupling mechanism ${ }^{37,56-59}$ giving rise to the intensity, while others have been concerned with the interpretation of the shapes ${ }^{55,59-61}$ of the exciton-magnon sidebands While the selection rules are independent of the particular coupling mecinanism, the band shapes are not, the latter thus providing an experimental check for theories of the nature of the coupling. Two different mechanisms have been proposed, each being a special case of the general theory due to Dexter ${ }^{62}$ for the simultaneous excitation of a pair of ions One approach ${ }^{37,56}$ involves a multipole expansion of the Coulomb interaction between pairs of ions, with emphasis on the coupling of the electric dipole moment of one ion to the spin-orbit induced electric quadrupole moment of the other ion The other approach (refs 57-59) is independent of spin-orbit coupling and involves instead exchange terms arising from the Coulomb interaction. Detaled studies of the closely related two-magnon spectrum of $\mathrm{MnF}_{2}$ suggest that the exchange mechanısm dominates in that case The spin-orbit-quadrupole mechanism involves mixing with even parity excited states which he only $1000-2000 \mathrm{~cm}^{-1}$ above the ground state for $\mathrm{Fe}^{\mathrm{II}}$ in $\mathrm{FeF}_{2}$, but approximately $20,000 \mathrm{~cm}^{-1}$ for $\mathrm{Mn}^{\mathrm{II}}$ in $\mathrm{MnF}_{2}$. Thus two-magnon intensities in $\mathrm{FeF}_{2}$ might be expected to be four orders of magnitude greater than for $\mathrm{MnF}_{2}$ (the energy denominator enters to the fourth power ${ }^{37,56}$ ). By contrast, the experimental intensities are comparable, which is compatible with the expectations of an exchange mechanism, this involving odd parity excited states occurring at similar energies for the two salts It seems reasonable to assume that this exchange mechanism is responsible for the cooperative intensification observed for many Mn II salts as well as for both like and unlike pairs of ions

It is important to note that the familiar Heisenberg scalar interaction

$$
H=J S_{\mathrm{A}} \cdot \boldsymbol{S}_{\mathrm{B}}
$$

where $S_{\mathrm{A}}$ is the total spin of ion $\mathrm{A}$ and $S_{\mathrm{B}}$ is the total spin of ion $\mathrm{B}$, cannot account for these observations as the above Hamiltonian not only commutes with $S^{2}$ and $M$, but also with $S_{\mathrm{A}}{ }^{2}$ and $S_{\mathrm{B}}{ }^{2}$. Thus the parr states have the total spin of each ion as good quantum numbers, which will not change during a radiative process unless spin-orbit coupling is present. If such couphng is present, so that $\Delta S_{\mathrm{A}}= \pm 1$, the pair selection rule becomes $\Delta S=0, \pm 1$, with observed parr spectra ${ }^{31,35,36,52}$ Instead we must approach ${ }^{57-59}$ the super-exchange coupling from the more fundamental equation 


$$
H=\sum_{i, j} J_{i j} s_{i \mathrm{~A}} s_{j \mathrm{~B}}
$$

where the summation is over electron pars, with

$$
s_{\mathrm{A}}=\sum_{i} s_{l \mathrm{~A}} \quad \text { and } \quad s_{\mathrm{B}}=\sum_{j} s_{i \mathrm{~B}}
$$

For ground-state ions, Hund's rule gives $S_{\mathrm{A}}=n_{\mathrm{A}} s_{1 \mathrm{~A}}$, where $n_{\mathrm{A}}$ is the number of magnetic electrons ion A Therefore eqn (31) can be rewritten

$$
H=\sum_{l, j} J_{y} \frac{1}{n_{\mathrm{A}} n_{\mathrm{B}}} S_{\mathrm{A}} \cdot S_{\mathrm{B}}=\left[\frac{1}{n_{\mathrm{A}} n_{\mathrm{B}}} \sum_{i, j} J_{y}\right] s_{\mathrm{A}} s_{\mathrm{B}}
$$

which serves as a definition of $J$ in eqn. (30) in terms of the individual contributions $J_{i j}$ which differ for different pairs of orbitals However, the Heisenberg form cannot be obtained from eqn (31) when one ion is in an excited state with a spm less than that of the ground state In such a case the coupling of the cons destroys the total spm of each ion as good quantum numbers The exchange-dependent electric dipole transition moment (refs $57-59$ ) is found to have a form similar to eqn. (31), with parameters containing information about odd parity excited states, such as charge-transfer states, which are necessary for the intensity mechanism

The remaining electric dipole process observed in salts such as $\mathrm{MnF}_{2}$ is the simultaneous excitation of two ions, also called two-exciton creation ${ }^{51-53}$ By analogy with eqns. (28) and (29) we have

$$
\begin{array}{ll}
\text { Sub-lattice A } S_{\mathrm{A}}=\frac{5}{2}, & M_{\mathrm{A}}=+\frac{5}{2}, S_{\mathrm{A}}^{\prime}=\frac{3}{2}, M_{\mathrm{A}}^{\prime}=+\frac{3}{2} \\
\text { Sub-ldttice B } S_{\mathrm{B}}=\frac{5}{2}, M_{\mathrm{B}}=-\frac{5}{2}, S_{\mathrm{B}}^{\prime}=\frac{3}{2}, M_{\mathrm{B}}^{\prime}=-\frac{3}{2}
\end{array}
$$

These excitations may also be called electronic overtones and combinations. The energies are elose to the appropriate sums of single exciton energies, if one is careful to subtract any magnon energies $\left(\sim 50 \mathrm{~cm}^{-1}\right)$ from the latter. Such transitions were first observed ${ }^{63}$ in the excitation spectrum of $\mathrm{Pr} I I I\left(4 f^{2}\right)$ doped in to $\mathrm{LaCl}_{3}$, and provided the stimulation for Dexter's general theoretical treatment ${ }^{62}$ of pair excitations Very similar interactions (refs 64,65 ) are observed in the spectrum of gaseous $\mathrm{O}_{2}$ at high pressures, as this molecule possesses $a$ half-filled $\pi_{g}{ }^{2}$ configuration giving rise to spin- and parity-forbidden absorptions from the ${ }^{3} \Sigma \bar{g}$ ground state

Many systems other than the simple fluorides have now been studied Detalled results for several hydrated $\mathrm{Mn}$ II salts including $\mathrm{CsMnCl}_{3} \cdot 2 \mathrm{H}_{2} \mathrm{O}, \mathrm{MnCl}_{2} 2 \mathrm{H}_{2} \mathrm{O}$, and $\mathrm{MnSO}_{4} \quad 4 \mathrm{H}_{2} \mathrm{O}$ have been reported by Marzzaco and $\mathrm{McClure}{ }^{66}$. Cooperative intensifications and pair excitations have been reported by Schugar et al. ${ }^{67-69}$ for binuclear Fe III complexes in aqueous solution. Gray ${ }^{70}$ reviews this and related work together with its 
biological implications The emission spectra of coupled $\mathrm{Cr}$ III and $\mathrm{Eu}_{\mathrm{u}}$ III Ions have been studied by van der Ziel and Van Uitert ${ }^{71}$, while Ferguson and Guggenheim report electrontransfer states of pars of unlıke transition metal ions in perovskite fluorides Dubicki and Martin have studied the binuclear acid and basic rhodo salts ${ }^{73}$ of $\mathrm{Cr}_{\mathrm{rII}}$, as well as the

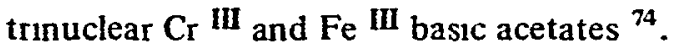

In summary it appears that whenever a species (free ion, diatomic molecule, or mononuclear complex) has very weak spin-and parity-forbidden electronic excitations, such as found, but not exclusively, with half-filled shells, that small or large aggregates of such species will display exchange-dependent spectral intensifications (break-down of Beer's law) together with the appearance of electronic overtone and combination bands It further seems reasonable to suggest that the latter represent the true nature of many transitions previously assigned as charge-transfer for the lack of a better explanation.

\section{REFERENCES}

1 For general revieus of the spectra of transition metal compleves, see (a) D S McClure, Solud State Phvs, 9 (1959) 399. (b) C K Jørgensen, Absorption Spectra and Chemical Bonding in Compleres Pergamon Press, Oxford, 1962 (c) N S Hush and R J M Hobbs, Progr Inorg Chem 10 (1968) 259, (d) A B P Lever, Inorganic Electronc Spectroscopy Elsevier, A nsterdam, 1968 (c) I Terguson, Progr Inorg Chem., 12 (1970) 159 For a collection of recent research papers, see (f H M Crosswhite and H W. Moos (Eds ), Optical Properties of Ions in Crystals, Interscience, New York, 1967

2 For a review of spectral intensities in transition metal complexes, see $\mathrm{C} J$ Ballhausen Progr Inorg Chem., 2 (1960) 251

3 For an earlier theorencal analysis of the intensities and Zeeman patterns of spin-forbidden transitions in compleses of Cr III, Mn II, etc, see S Sugano, Progr Theor Phys Suppl No 14 (1960) 66

$4 \mathrm{C}$ K. Jorgensen, Absorption Spectra and Chemucal Bonding in Compleves Pergamon Press, Oxtord, 1962, p 92

5 I S Griftith, The Theory of Transition Metal Ions Cambndge University Press New York. 1961 pp. 45-57, ?89-292.

$6 \mathrm{~L} \mathrm{~J} \mathrm{~F} \mathrm{Broer,} \mathrm{C} \mathrm{J} \mathrm{Gorter} \mathrm{and} \mathrm{J} \mathrm{Hoogschagen,} \mathrm{Physica} \mathrm{(Utrecht).} 9$ (1945) 231

7 C K. Jorgensen and B R Judd, Mol Phys 8 (1964) 281

8 For an evample of spin-allowed magnetic dipole transtions in octahedral $N_{1}$ II compleses, sce J Ferguson, H J Guggenheim, L F. Johnson and H Kamımura, J Chem. Phys, 38 (1963) 2579

9 For the magnetic dipole spectrum of Mn $\mathrm{Si}_{6} 6_{6} \mathrm{O}$, see 1. Tsujıkawd, J Phirs Soc Jap 18 (1963) 1391

10 For the magnetic dipole spectrum of $\mathrm{MnI}_{2}$ see $\mathrm{R} S$ Meltzer and $\mathrm{L} \mathrm{L}$ Lolur, Jr $J$ Chem Phys 49 (1968) 541 and references therein

11 For a review oriented toward singlet-triplet transitions in heterocyche molecules, see $L$ Goodman and B.J. Laurenz1, Advan Quantum Chem. 4 (1968) 153.

12 See, for example, J S. Griffith, The Theory of Transinon Metal lons Cambridge University Pres, New York, 1961, pp. 106-113, 240-244,

13 M Mizushima, Phys. Rei. A, 134 (1964) 883

14 The parameter $\xi$ increases steeply with atomic number $Z$, for hydrogenic atoms with quantum numbers $n$ and $l$,

$$
\zeta_{n, l}=\frac{e^{2} \hbar^{2} Z^{4}}{2 m^{2} c^{2} n^{3} a_{0}{ }^{3} l\left(l+\frac{1}{2}\right)(l+1)}
$$

where $a_{0}$ is the Bohr radius $\hbar^{2} / m e^{2}$ See C.U Condon and G.H. Shortley, The Theory of Atomic Spectra, Cambridge Unversity Press, London, 1963, pp 120-125 For many-electron atoms the $Z$ dependence is not so steep

Coord. Chem. Rev., 8 (1972) 
15 U Blume and R.E Watson, Proc Ro, Soc, Ser A 270 (1962) 127 and 271 (1963) 565 For derivations of equ (9), yee $H A$ Bethe and L. C Salpeter, Quantum Mechancs of One-and TwoElectron Atoms Acdutemic Press New York 1957 pp 170-205 and J C Slater, Quantum Theory of Atomic Structure Vol II McGraw-Hill, New York, 1960, pp I89-200

16 H Horie, Progr Theor Phys 10 (1953) 296

$17 \mathrm{~L} \mathrm{~L} \mathrm{Lohr,} \mathrm{Jr.J.} \mathrm{Chem} \mathrm{Phys.} 45$ (1966) 1362 We have also shown tor systems with spin-orbit coupling of the form in eqn (10) that the magnetic dipole operator should contain, in addition to $L+2 S$, the additional term $\left(2 m c^{2}\right)^{-1} \Sigma_{2} \times\left(s_{2} \times \operatorname{grad}_{2} V\right)$ If $V=-\Sigma Z e^{2} / r_{1}$, the term becomes $\left(Z c^{2} / 2 m c^{2}\right)=\left(r_{2} \times\left(s, \times r_{l}\right)\right) / r_{l}^{3}$ In either form this small term can directly connect states of the same paity but diftering spin thus contributing to the spin-forbidden magnetie dipole intensity No new term is found to this order for electric quadrupole processes

I8 R Englman $J$ Chcm Phis 45 (1966) 2669

19 Y N Chiu, J Chem Phy's 48 (1968) 3476

20 Note that the size of part $(d)$, corresponding to the spin-torbiaden magnetic dipole term in ref 17 , 1s about $4 \%$ of the indirect, even parity contribution

21 Each "size" is in Lgs units such that multiplication by $\nu / h c$ yields the transition probability as the Golden Rule " number rate For the meaning ot the latter, sec, tor elample, $\mathrm{C}$ Merzbacher

Quantum Mechancs Wiley, New York 1961, pp. 439-481

22 T U Dunn and WK Lea, $J$ Chem Phys 46 (1967) 2907

23 D S McClure $J$ Chcm Phins 17 (1949) 905

$24 \mathrm{H} \mathrm{I}$ Hameka and L Oosterhott, Mol Phl's 1 (1958) 358

$25 \mathrm{~L} \mathrm{H}$ Aller, C W Uttord and J H Van Vluck Astropliy's $J 109$ (1949) 42

26 See lor wample P W Anderson Soltd State Phy's 14 (1963) 99

27 A L Scluawlow, D C Wood and A M Clogston, Phy's Rel Lett, 3 (1959) 271

28 D S McClurt $J$ Chcm Phys, 39 (1963) 2850

29 S lbuki and D Langer $J$ Phis Soc Jap 19 (1964) 422

30 D Langer and S lbuki, Phls Rel'A 138 (1965) 809.

$31 \mathrm{~J}$ Ferguson $11 \mathrm{~J}$ Guggenhcim and $Y$ Tanabe, $J$ Appl Phl's, 36 (1965) 1046

$32 \mathrm{~L} \mathrm{~L}$ Lohr, $\mathrm{Jr}$ and DS McClure, $J$ Chem Plyss, 49 (1968) 3516

$33 \mathrm{~J}$ Terguson and II J Guggenhem, J Chem Phy's, 44 (1966) 1095

$34 \mathrm{~J}$ Ferguson, If J Guggenhem and Y Tanabe, Phys. Rev. Lett, 14 (1965) 737

$35 \mathrm{~J}$ Ferguson, $11 \mathrm{~J}$ Guggenhem and $Y$ Tanabe $J$ Chem Phis 45 (1966) 1134

$36 \mathrm{~J}$ Ferguson, H J Guggenhicim and Y Tanabe, Phys Rel , 161 (1967) 207.

$37 \mathrm{~J}$ W. Halley and I Silvera, Phys Rel Lett, 15 (1965) 654

38 I Silvera and J W. Halley, Phy's Rel 149 (1966) 415

39 J.W Halley Phis Rel, 149 (1966) 423, 154 (1967) 458

$40 \mathrm{~S} J$ Allen, $\mathrm{J}_{\mathrm{r}} \mathrm{R}$ Loudon and $\mathrm{PL}$ Richards, Ply's Rel Lett, 16 (1966) 463

41 R Loudon, Adian Phys, 17 (1968) 243

42 R L Grecne D D Sell. W M Yen A L Schawlow and R M White, Plys Rev. Lett, 15 (1965)656

43 r Tanabe T. Moriya and S Sugano, Phis Reı. Lett, 15 (1965) 1023

44 R Stevenson, Can J Phis 43 (1965) 1732

45 R Stevenson, Phy's Rct 152 (1966) 531

46 R C. Dietz, A Misetich and H J Guggenheim, Phys Ret Lett, 16 (1966) 841

47 P G Russell. D S McClure and J.W Stout, Phiss Rel Lett, 16 (1966) 176

48 D S McClure, R Meltzer, S A Reed and J WV Stout, in H M Crosswhite and H WV Moos (Eds),

Optical Properties of lons in Crystals Interscience, New York, 1967

49 D D Sell, R L Greenc and R M Whitc, Phws Rer 158 (1967) 498

50 D D Sell, J Appl Phrs 39 (1968) 1030

51 J Ferguson, Aust J Chem 21 (1968) 307

52 J I van der Ziel, Phys Rct. Lett 26 (1971) 766.

53 S C Stokowshi and D D Sell, Phys Rev B, 3 (1971) 208

54 S Washimiya and K I Gondaira, J Phys Soc Jap. 23 (1967) 1

55 R Maltzer, $M$ Lowe and D S. McClure, Phus. Rel. 180 (1969) 561 
S6 J W Halley, Phys Rev 144 (1966) 423.

57 K.I Gondara and Y. Tanabe, J Phys. Soc. Sap 21 (1966) 1527

58 Y. Tanabe and K I Gondaird, J. Phys Soc. Jap. 22 (1967) 573

59 Y. Tandbe, K.I. Gondaira and H Murata, $J$ Phys Soc Jap, 25 (1968) 1562.2.

60 S. Frceman and J.J. Hopfield, Phys Rev, Lett, 21 (1968) 910

61 R S Meltzer, M Y Chen, D S McClure and M Lowe-Parıseau, Phys Reı Lett, 21 (1968) 913.

62 D L Dexter, Phys Rev, 126 (1962) 1962

63 F. Varsanyi and G.H. Dieke, Phys Rev. Lett., 7 (1961) 442.

64 V G Krishna, J. Chem. Phys, 50 (1969) 792.

65 O L J Gızeman, E A. Ogryzlo and R.P H Rettschnick, J Chem. Phys, 52 (1970) 3718

66 C. Marzzacco and D S. McClure, Symp. Faraday' Soc , 3 (1969) 106

67 H. Schugar, C. Walling, R.B. Jones and H B Gray, J Amer Chem. Soc, 89 (1967) 3712.

68 H.J Schugar, A T. Hubbard, F.C. Anson and H B Gray, J. Amer Chem Soc, 91 (1969) 71

$69 \mathrm{H} \mathrm{J}$ Schugar, G R Rossman, J Thibeault and H B. Gray, Chem Phys Lett . 6 (1970) 26

70 H B Gray, Biolnorganic chemistry, Amer Chem. Soc Monogr 100, 1971, p 365

71 J P van der Ziel and L G Van Uitert, Phys Rev, 180 (1969) 343, I 86 (1969) 332

72 J. Ferguson and H I Guggenhem, Phys Rev $B, 1$ (1970) 4223

73 L. Dubickı and R L Martin, Aust $J$ Chem., 23 (1970) 215

74 L Dubickı and R.L Martin, Aust $J$ Chem, 22 (1969) 701

Coord. Chem. Rev, 8 (1972) 